\title{
Pulsating emission of droplets from an electrified meniscus
}

\author{
F.J. Higuera ${ }^{a, *}$, S.E. Ibáñez ${ }^{a}$, A.J. Hijano ${ }^{b}$, I.G. Loscertales ${ }^{b}$ \\ ${ }^{a}$ E.T.S. Ingenieros Aeronáuticos, UPM, Plaza Cardenal Cisneros 3, 28040 Madrid, Spain \\ b Escuela de Ingenierías, Universidad de Málaga, Calle Dr. Ortiz Ramos, 29071 Málaga, Spain
}

\begin{abstract}
A numerical description is given for the pulsating emission of droplets from an electrified meniscus of an inviscid liquid of infinite electrical conductivity which is injected at a constant flow rate into a region of uniform, continuous or time periodic, electric field. Under a continuous field, the meniscus attains a periodic regime in which bursts of tiny droplets are emitted from its tip. At low electric fields this regime consists of sequences of emission bursts interspersed with sequences of meniscus oscillations without droplet emission, while at higher fields the bursts occur periodically. These results are in qualitative agreement with experimental results in the literature. Under a time periodic electric field with square waveform, the electric stress that acts on the surface of the liquid while the field is on may generate a tip that emits tiny droplets or may accelerate part of the meniscus and lead to a second emission mode in which a few large droplets are emitted after the electric field is turned off. Conditions under which each emission mode or a combination of the two are realized are discussed for low frequency oscillatory fields. A simplified model is proposed for high electric field frequencies, of the order of the capillary frequency of the meniscus. This model allows computing the average emission rate as a function of the amplitude, duration and bias of the electric field square wave, and shows that droplet emission fails to follow the applied field above a certain frequency.
\end{abstract}

\section{Introduction}

Ink-jet printing based on electrohydrodynamic atomization is attracting much attention as a possible alternative to established thermal and piezoelectric techniques. This has led to a renewed interest in the pulsating emission of droplets from an electrified meniscus, which is the subject of this paper. Among the salient features of the new technique are its ability to generate droplets much smaller than the diameter of the injection nozzle, whose volume may be changed without changing the nozzle, and the simplicity of the nozzles required, while its main limitations in its present state are its low speed and the relatively large size dispersion of the droplets produced. Droplet-to-nozzle diameter ratios down to about $1: 5$ and 1:15 have been reported by Chen et al. (2006) $(10 \mu \mathrm{m}$ droplets of an aqueous solution issuing from a $50 \mu \mathrm{m}$ i.d. nozzle) and by Lee et al. (2012) (50 $\mu \mathrm{m}$ droplets of diethylene glycol issuing from a $840 \mu \mathrm{m}$ i.d. nozzle), respectively. For comparison, the diameters of the droplets produced with the piezoelectric technique are often the same as the diameter of the printer's nozzle, though diameter ratios smaller than $1: 2$, with volume variations by a factor of 10 , have been reported by Chen $\&$ Basaran (2002) when the time scales that govern the flow in the nozzle are chosen to replace emission of the forming droplets by emission of tongues protruding from these droplets; see Basaran et al. (2013) for a recent review.

\footnotetext{
* Corresponding author.

E-mail address: fhiguera@aero.upm.es (F.J. Higuera).
} 
The characteristics of EHD printing make this technique suitable for a variety of applications in material processing and the life sciences, such as nano-printing or patterning for manufacturing semiconductors (Sirringhaus et al., 2000), visual displays (Hebner et al., 1998) and solar cell electrodes, micromechanical devices (Cheng et al., 2005; Jaworek \& Sobczyk, 2008), particle production and encapsulation in the pharmaceutical industry (Yurteri et al., 2010), biosensors (Park et al., 2007, 2008), thin film deposition (Miao \& Xiao, 2002), and research in biology (Jayasinghe \& Townsend-Nicholson, 2006).

Electrohydrodynamic atomization relies on electrical stresses to atomize a liquid. These stresses arise at the surface of a meniscus of the liquid to be atomized, which must be an electrical conductor, when a voltage is applied between the nozzle through which the liquid is injected and another electrode holding the substrate onto which the droplets are deposited; see Fernández de la Mora (2007) for a review. Cloupeau \& Prunet-Foch (1994) and Jaworek \& Krupa (1999) gave classifications of the functioning modes of an electrospray. Juraschek \& Rollgen (1998) found that the system displays two pulsating and one continuous axial spray modes when the applied DC voltage is increased keeping the flow rate constant. In axial spray mode I, at lower voltages, mass and electric current emission occur in sequences of bursts of droplets interspersed with intervals of no emission, in a cycle that repeats itself with a large period compared to the duration of individual bursts. The time interval between sequences of bursts decreases when the applied voltage increases, until the system transitions to axial mode II, in which bursts occur with a constant frequency that increases with the applied voltage. Finally the system transitions to a cone-jet mode of continuous emission when the voltage is further increased. Juraschek and Rollgen (1998) explain their pulsating modes, and the existence of two frequencies in mode I, in terms of an imbalance between the rate of liquid emission and the rate at which the liquid is supplied to a small region around the tip of the meniscus or to the whole meniscus.

More recent experiments with DC voltages have revealed many complexities, but a quantitative theory is still missing. Thus, on the basis of a limited data set, Alexander et al. (2006) reported only mode II for unforced electrosprays (electrosprays for which the flow rate is not imposed but determined by the electric suction at the liquid surface) when the applied voltage is increased past the onset of current oscillation. Paine et al. (2007) noted that not all the liquids show the same pulsation nature across the range of applied voltages wherein stable pulsating modes can be observed. They found mode I in unforced electrosprays of low conductivity water and ethylene glycol solutions with large nozzles, but not with triethylene glycol solutions. Alexander et al. (2006) also found a new mode that they termed as mode IIb in which the variation of the time average current with voltage is different from that of mode II, and they noted that the evolution of the pulsation frequency and the pulse duration with voltage depends on the conductivity of the liquid and the diameter of the nozzle. Paine et al. (2007) concluded that mode IIb can only be expected to occur in situations where increasing the voltage causes the liquid meniscus to retract. Mode I is also absent in some forced electrosprays (Hijano et al., 2012). Vertes and coworkers used a combination of current measurements and fast images, together with dynamical systems theoretical concepts, to study the axial modes of an electrospray and the transitions between different electrospray regimes. They proposed a classification of all the observed axial regimes of electrospray in terms of only three regimes, dripping, pulsating and cone-jet, potentially separated by two chaotic regimes, burst and astable, respectively; see Marginean et al. (2007) and references therein. In this classification, the burst regime amounts to axial mode I of Juraschek \& Rollgen (1998), and is viewed as a transition between the dripping and the pulsating regimes, the latter of which amounts to axial mode II. Similarly, the astable regime, not to be discussed below, is a transition between the pulsating and the cone-jet regimes, which is an alternative route to the direct transition between them. Marginean et al. (2006) correlated their current oscillation frequency versus contact line radius measurements with Rayleigh's expression for the natural frequencies of an isolated electrically charged droplet, and used these results to estimate the charge of a pulsating meniscus in terms of the spray current oscillation frequency. Other attempts at finding scaling laws for the pulsating modes of a system subject to DC voltage have been reported by Chen et al. (2006) and Choi et al. (2008).

Some of the pulsating modes of an electrospray are better suited for printing applications than continuous, cone-jet emission, though the latter has also been used (Lee et al., 2007). The pulsating modes discussed above, which are obtained with a DC voltage, can be used for printing, sometimes giving uniform droplet volumes and velocities; see, e.g., Jayasinghe et al. (2002), Wang et al. (2005), Paine et al. (2007), and Park et al. (2007). However, Chen et al. (2006), Mishra et al. (2010) and Lee et al. (2012), among others, find that these modes do not allow simultaneous control of the size of the deposited droplets and the printing frequency, and that they are sensitive to perturbations leading to inconsistent droplet array properties. Seeking more flexible control, Kim et al. (2008) used a square wave pulsed voltage superimposed on a DC voltage bias, and showed that the frequency of droplet generation can be locked on to the frequency of the applied voltage when the latter is sufficiently small, while emission fails to occur in some cycles of the applied voltage when its frequency increases. Although a quantitative model of the response of an electrospray to a time periodic applied voltage, or a classification of the functioning modes analogous to that mentioned above for a DC voltage, is not available, Kang et al. (2011) and Lee et al. (2012) identified four droplet ejection modes in their experiments with various voltage amplitudes and durations, and found that the mode that they term microdripping, in which one or a few individual droplets are ejected from the tip of the meniscus per cycle of the applied voltage, is best suited for printing applications. This mode, however, is obtained only at low voltage frequencies and in a narrow region of the amplitude-duration plane.

The possibility of high speed printing with droplet emission driven by an electric field that pulsates at a frequency of the order of the capillary frequency of the meniscus has received considerable attention recently. Mishra et al. (2010) claim that appropriate choices of the peak and bias voltages lead to a fast jetting regime which allows high printing speed, in the order of 1000 droplets per second, with the volume of the droplets collected on the substrate determined by the duration of the 
voltage pulses. These authors proposed rules for shaping the voltage signal, which are scarce in the literature; see also Sutanto et al. (2012) and references therein. Pressure and gravity feeding systems were used that only deliver liquid when the electric stress induces a depression in the meniscus that overcomes surface tension, an idea that originated with unforced nanoelectrosprays; see Paine (2009) and references therein. Analysis of some of these systems by Stachewicz et al. (2009a,b) approximately determines the lower and upper limits of voltage pulse duration, and the upper limit of pulse frequency, within which regular single event electrospray pulses are possible. These analyses also show a strong coupling between the feeding system and the flow in the meniscus.

This paper presents a numerical investigation of the pulsating emission of droplets from an electrified meniscus, aimed at improving our understanding of the dynamics of the flow under DC and pulsed electric fields. The numerical computations determine the distributions of electric field and electric stress acting on the surface of the liquid, which are central to the droplet emission process but are virtually impossible to measure in experiments, as well as the ensuing evolution of the liquid and its surface. The numerical results capture and allow one to explain behaviors observed in experimental studies, some of which are mentioned in the following paragraph, and include predictions verifiable in future experiments. The model assumes that an inviscid liquid of infinite electrical conductivity is injected at a constant flow rate through an orifice in a planar electrode into a region of uniform electric field, induced by a high continuous or time periodic voltage applied between this electrode and a far parallel electrode. To leave out the viscosity of the liquid may be a reasonable approximation for experiments carried out with aqueous solutions, methanol, or ethanol (e.g. Chen et al., 2006; Choi et al., 2008; Juraschek \& Rollgen, 1998; Kang et al., 2011; Marginean et al., 2006; Mishra et al., 2010), though probably not for experiments with glycerol or ethylene glycol (Choi et al., 2008; Kim et al., 2008; Lee et al., 2012). The effect of the finite electrical conductivity of the liquid has been investigated by Kang et al. (2011). Provided it is sufficiently high, the conductivity does not seem to have the important role it plays in the cone-jet mode (Fernández de la Mora, 2007; Fernández de la Mora \& Loscertales, 1994).

The numerical results of Section 3 show that the response of the meniscus to a DC electric field becomes time periodic after a long initial transient. These results reproduce axial modes I and II of Juraschek \& Rollgen (1998), depending on the value of the field, and suggest an explanation for the quenching of droplet emission bursts. The response of the meniscus to a time periodic square-wave electric field with a frequency that is small compared to the meniscus capillary frequency is examined in Section 4, where two different modes of emission are found. The pulsed electric field may either cause the formation of a tip from which many tiny droplets are emitted while the field is on, much as for a DC field, or it may cause an acceleration of the liquid that leads to the emission of a few larger droplets after the field is turned off. The second mode is akin to the microdripping mode of Kang et al. (2011) and Lee et al. (2012). The conditions under which each mode, or a combination of the two, is obtained are discussed. A preliminary study is given in Section 5 of the response of the meniscus to an electric field oscillating at a frequency of the order of the meniscus capillary frequency. Additional simplifying assumptions are used to approximately determine conditions under which droplet emission locks on to the oscillating field.

\section{Formulation}

The configuration sketched in Fig. 1 will be used as a model of the real droplet generation process. A flow rate $Q$ of an inviscid liquid of density $\rho$, surface tension $\gamma$, and infinitely high electrical conductivity is injected into the space between two plane parallel electrodes through an orifice of radius $a$ in one of the electrodes. The distance between the electrodes is large compared to $a$, and a high voltage is applied between them that induces a uniform electric field $E_{\infty}$ away from the orifice.

The surface of the liquid is an equipotential of the electric field, and the presence of the liquid perturbs the uniform field $E_{\infty}$ in the vicinity of the orifice. The electric potential $\varphi$ is to be found by solving the Laplace equation $\nabla^{2} \varphi=0$ outside the liquid with the boundary conditions $\rho=0$ at the surfaces of the liquid and the electrode through which the liquid is injected, and $-\nabla \varphi=E_{\infty} \boldsymbol{e}_{x}$ for $x \rightarrow \infty$, where $x$ is the distance along the axis of the orifice, measured from the electrode, and $\boldsymbol{e}_{x}$ is a unit vector in the direction of this axis.

The flow of the liquid is irrotational. The velocity potential $\phi$, such that $\boldsymbol{v}=\nabla \phi$, satisfies the Laplace equation in the liquid with homogeneous Neumann conditions at the wall of the injection orifice $(r=a, x<0) ; \boldsymbol{e}_{x} \cdot \nabla \phi=Q / \pi a^{2}$ for $x \rightarrow-\infty, r<a$; and conditions at the free surface of the liquid which will be discussed below. The contact line of the liquid with the electrode is taken to coincide with the edge of the injection orifice $(x=0, r=a)$. Here $r$ is the distance to the axis of the orifice. The pressure of the liquid satisfies the Euler-Bernoulli equation $\partial \phi / \partial t+\frac{1}{2}|\nabla \phi|^{2}+p / \rho=C(t)$ (see, e.g., Batchelor, 1967), where the effect of the gravity has been left out and the value of $C(t)$ is determined by imposing the additional condition $\phi=0$ at the contact line.

The surface of the liquid, say $f(\boldsymbol{x}, t)=0$ with $f<0$ in the liquid, is a free material surface to be found as part of the solution. The kinematic condition $D f / D t=0$, where $D / D t=\partial / \partial t+\boldsymbol{v} \cdot \nabla$, and the balance of stresses $p+\frac{1}{2} \varepsilon_{0}|\nabla \varphi|^{2}=\gamma \mathcal{C}$ are to be satisfied at this surface. Here and above $p$ is the pressure of the liquid referred to the pressure of the surrounding medium, which is assumed to be a dielectric of permittivity $\varepsilon_{0}$ playing no role in the dynamics (a gas or a vacuum), and $\mathcal{C}=\nabla \cdot \boldsymbol{n}$, with $\boldsymbol{n}=\nabla f /|\nabla f|$, is twice the mean curvature of the surface. The second term on the left-hand side of the balance of stresses is the electric stress at the equipotential surface of the liquid (see, e.g., Landau \& Lifshitz, 1960), and the term on the right-hand side is the stress due to the surface tension. 

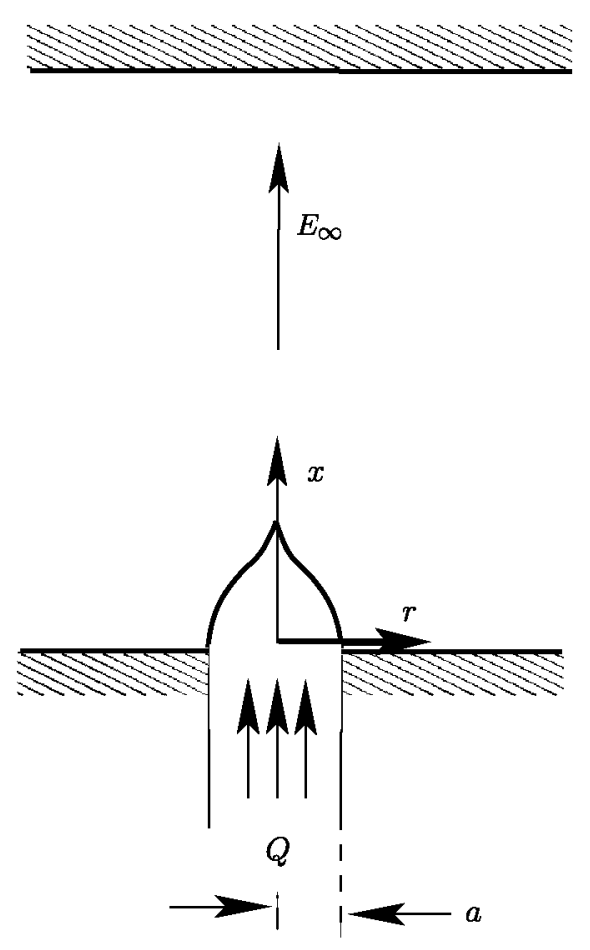

Fig. 1. Definition sketch.

In the limit of infinitely high electrical conductivity considered here, the surface charge density immediately adjusts to its local equilibrium value $-\varepsilon_{0} \nabla \varphi \cdot \boldsymbol{n}$ which screens the liquid from the outer electric field. The dynamics of the surface charge is infinitely fast compared to the dynamics of the liquid under the electric and surface tension stresses acting on its surface, and needs not be analyzed in detail as it plays no role in the evolution of the meniscus.

In what follows, distances are scaled with the radius of the orifice, the velocity and pressure of the liquid with the capillary velocity $v_{0}=(\gamma / \rho a)^{1 / 2}$ and $\rho v_{0}^{2}$, respectively, times with the capillary time $t_{0}=a / v_{0}$, the electric field with $E_{0}=\left(\gamma / \varepsilon_{0} a\right)^{1 / 2}$, and the electric potential with $E_{0} a$. The scales of velocity and electric field are suggested by the balance of pressure, electric and surface tension stresses at the surface of the liquid, with $p=O\left(\rho v_{0}^{2}\right), \frac{1}{2} \varepsilon_{0}|\nabla \varphi|^{2}=O\left(\varepsilon_{0} E_{0}^{2}\right)$ and $\mathcal{C}=O(1 / a)$; i.e., $\rho v_{0}^{2}=\varepsilon_{0} E_{0}^{2}=\gamma / a$.

The condition above, that the liquid is effectively inviscid and the flow is irrotational, relies on the assumption that the Reynolds number $O h^{-1}=\rho v_{0} a / \mu=(\rho \gamma a)^{1 / 2} / \mu$, where $\mu$ is the viscosity of the liquid, is high. This is the case in many experiments carried out with aqueous solutions, methanol or ethanol; for example, $O h^{-1}=28$ for water with $a=1 \mathrm{~mm}$. The condition that the surface of the liquid be an equipotential is satisfied when the electrical relaxation time $t_{e}=\varepsilon_{0} \varepsilon / K$, where $\varepsilon$ and $K$ are the dielectric constant and the conductivity of the liquid, respectively, is small compared to the characteristic mechanical time. The ratio $t_{e} / t_{0}=2 \times 10^{-6}$ for water with $K=0.1 \mathrm{~S} / \mathrm{m}$ and $a=1 \mathrm{~mm}$.

Denoting the dimensionless variables with the same symbols used before for their dimensional counterparts, the governing equations take the form

$$
\nabla^{2} \varphi=0
$$

outside the liquid, for $f(\boldsymbol{x}, t)>0$,

$$
\nabla^{2} \phi=0
$$

in the liquid, for $f(\boldsymbol{x}, t)<0$,

$$
\begin{aligned}
& \varphi=0, \\
& \frac{D \phi}{D t}=\frac{1}{2}|\nabla \phi|^{2}+\frac{1}{2}|\nabla \varphi|^{2}-\nabla \cdot \boldsymbol{n}+C(t), \\
& \frac{D f}{D t}=0
\end{aligned}
$$

at the surface of the liquid, $f(x, t)=0$, and

$$
\varphi=0 \quad \text { at } x=0, \quad r>1
$$




$$
\begin{aligned}
& -\nabla \varphi=E_{\infty} \boldsymbol{e}_{x} \quad \text { for } x \rightarrow \infty \\
& \frac{\partial \phi}{\partial r}=0 \quad \text { at } r=1, x<0 \\
& \nabla \phi=\frac{q}{\pi} \boldsymbol{e}_{x} \text { for } r<1, x \rightarrow-\infty \\
& \phi=f=0 \quad \text { at } x=0, r=1,
\end{aligned}
$$

where (3b) is the Euler-Bernoulli equation at the surface of the liquid, with the pressure eliminated by using the balance of stresses. The far dimensionless field in (5) is assumed to be a time periodic field of the form

$$
E_{\infty}= \begin{cases}E_{p} & \text { for } \bmod (t, T)<t_{d} \\ E_{b} & \text { for } \bmod (t, T)>t_{d},\end{cases}
$$

which is a square wave of dimensionless period $T$ with the peak value $E_{p}$ during a duty time $t_{d}$ per period, and the bias value $E_{b}<E_{p}$ during the rest of the period. Problem (1)-(9) depends on the five dimensionless parameters

$$
q=\frac{\rho^{1 / 2} Q}{\gamma^{1 / 2} a^{3 / 2}}, \quad E_{p}, \quad E_{b}, \quad t_{d}, \quad T,
$$

where the dimensionless flow rate $q$ is the square root of a Weber number, the peak and bias electric fields are scaled with $E_{0}$, and the times $t_{d}$ and $T$ are scaled with the capillary time $t_{0}$.

Axisymmetric solutions of (1)-(9) have been computed using standard boundary elements methods to solve the Laplace equations (1) and (2), and a second order Runge-Kutta method to advance the evolution equations (3b) and (3c) in time. Further details of the numerical method can be found in Higuera (2004, 2007).

Detachment of droplets from the meniscus is a discontinuous process that involves the formation and pinchoff of a ligament and a neck. Here no attempt is made to accurately compute the last stages of this process. Instead, as in the previous work (Higuera, 2007), detachment is assumed to occur when the radius of the neck becomes smaller than a certain cutoff, which was varied in the range from $2 \times 10^{-2}$ to $2 \times 10^{-3}$. Then the detaching droplet is removed and the tip of the meniscus is rounded with a spherical cap to continue the computation. The results are not very sensitive to the value of the cutoff, but it is clear that the procedure involves a numerical artifact. Thus, in the computations of the following section, the surface tension stress is small compared to the electric stress in the region of the neck where the cutoff radius is attained (compare the solid and dashed curves in each panel of Fig. 4, which give the distributions of normal electric and surface tension stresses along the surface of the liquid), while the surface tension is expected to play a role in real detachments (possibly along with effects of finite liquid conductivity and viscosity, which are not included in the model). It seems, however, that the fate of the shrinking neck is decided at the time its radius reaches the cutoff value, which justifies the numerical procedure; see Higuera (2007) for further comments.

\section{DC electric field}

A DC electric field is obtained by setting $E_{b}=E_{p}$ in (9), so that the parameters $t_{d}$ and $T$ drop out of the problem and the solution depends only on the dimensionless parameters $q$ and $E_{\infty}=E_{p}=E_{b}$. Computations carried out for various sets of values of these parameters show that the solution settles to a permanent, apparently periodic regime after a transient that depends on the initial conditions and may be fairly long. This transient will not be discussed here, but it should be mentioned that transient effects are very disturbing when using electrohydrodynamic atomization for printing applications. Permanent solutions are presented for $q=0.1$ and different values of $E_{\infty}$. Solutions for smaller values of $q$ are qualitatively similar but take longer computational times.

The numerical results show that two processes with very different time scales coexist in the evolution of the meniscus. The slow process is an oscillation of the whole meniscus or of a substantial portion of it, which occurs continuously for the inviscid liquid assumed here. The fast process is a rapid elongation of a small region around the tip of the meniscus that leads to the emission of tiny droplets. Emissions occur in bursts, with the number of droplets emitted per burst and the interval between bursts depending on the flow rate and the applied electric field.

The liquid inertia and surface tension play a role in the meniscus oscillation, so that the characteristic time of the slow process is the capillary time (unity in dimensionless variables). The fast process has been described elsewhere (Higuera, 2007). It is dominated by the self-intensification of the electric field when the equipotential surface of the liquid elongates into a filament. The electric field increases with streamwise distance along the filament in qualitative agreement with the predictions of slender body theory (Hinch, 1991). This leads to large electric stresses that overcome surface tension in a long region around the end of the filament, where the liquid is accelerated. The filament is thus stretched until pinchoff eventually occurs near its end and a droplet is emitted. The process is largely autonomous. A certain elongation of the meniscus is needed to start the intensification of the electric field, but once it is triggered, the self-intensification and stretching proceed independent of the evolution of the rest of the meniscus. 


\subsection{Mode I}

Figure 2 shows a period of the meniscus evolution for $E_{\infty}=0.5$. It comprises several stages. First the meniscus oscillates without emission of droplets (insets $\mathrm{A} 1-\mathrm{A} 4$ ), so that its volume increases linearly with time due to the injected flow rate. Due to this increase, the maximum curvature of the tip, at the instants of maximum elongation, increases with successive oscillations until the fast process mentioned above is triggered for the first time at about $t=14.3$ (marked by a black circle in Fig. 2). This leads first to the emission of a few droplets per oscillation, and then to the emission of a larger number of droplets before the tip recedes (insets B1 and B2). This stage is followed by the formation of a thick filament resembling a spindle (see the classification of Cloupeau \& Prunet-Foch, 1994), whose tip continuously emits droplets and whose base eventually develops a neck (insets $\mathrm{C} 1$ and $\mathrm{C} 2$ ). The spindle detaches from the meniscus (inset D) and droplet emission ceases at $t=26.88$, which is marked by a second black circle. The whole cycle repeats with a period of about 26.88 . The sequences of emission bursts separated by intervals of no emission resemble mode I of Juraschek \& Rollgen (1998), to the extent that this regime can be captured by the present model of inviscid infinitely conducting liquid.

Juraschek and Rollgen did not report additional large droplets at the end of a sequence of tiny droplet bursts, which results when a spindle detaches. However, this detachment can be seen in the high speed images of Marginean et al. (2006) and in our own experiments (Hijano et al., 2012). It seems to be the means by which the imbalance between emission and supply of liquid to the meniscus, hypothesized by Juraschek and Rollgen (1998) to cause mode I, is brought about in the cases considered here.

Periodic solutions for $E_{\infty}=0.3$ and 0.6 are qualitatively similar to the one described above. The period of the solution increases when $E_{\infty}$ decreases, from about 20 for $E_{\infty}=0.6$ to about 100 for $E_{\infty}=0.3$. The maximum volume of the meniscus also increases with decreasing $E_{\infty}$, from 2 for $E_{\infty}=0.6$ to 2.6 for $E_{\infty}=0.5$ and to 10 for $E_{\infty}=0.3$, and the sequence of events comprising a cycle becomes more complex and with higher cycle-to-cycle variability. Thus, when $E_{\infty}=0.3$, many droplets are shed per oscillation already at the onset of emission, and the shape of the spindle in the final part of the cycle may be more complex than in Fig. 2, with various successive detachments.

\subsection{Mode II}

A continuous sequence of emission bursts is obtained when $E_{\infty}=0.7$; see solid curves in Fig. 3 . In this case the meniscus elongates without prominent oscillations until a spindle develops and begins to emit tiny droplets. Many droplets are sequentially emitted in a certain lapse of time, during which the rapid back and forth displacement of the tip leads to the blurred patches in Fig. 3(b). The average length of the spindle increases with time, despite the continuous emission of droplets. At some point, a neck forms at the base of the spindle that slowly narrows until pinchoff occurs and the spindle detaches from the meniscus. A single detachment occurs per cycle, which leaves behind a small meniscus that cannot intensify the applied electric field, so that emission ceases until the volume increases and the cycle repeats. The period of the emission cycle is about 1.17 , but there is some variation of the volume of the meniscus at the end of emission over

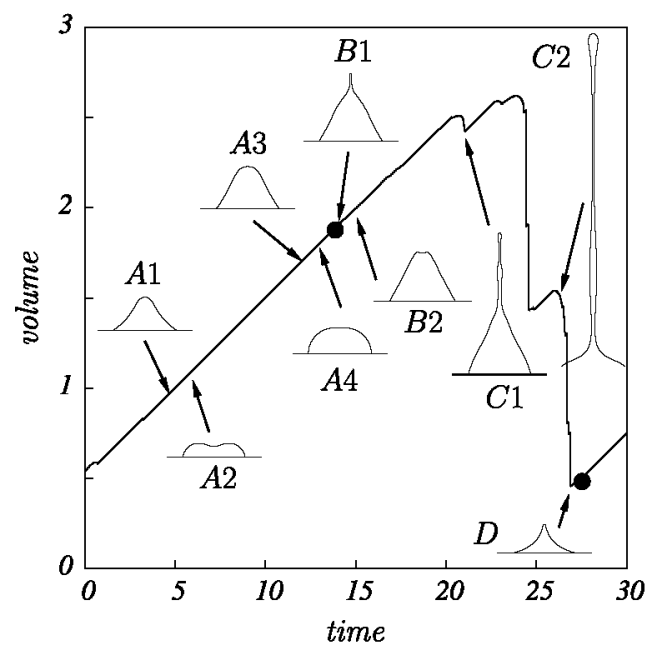

Fig. 2. Dimensionless volume of the meniscus as a function of dimensionless time during a cycle of the meniscus evolution for $q=0.1, E_{\infty}=0.5$. The insets show the shape of the meniscus at different instants during the cycle. Insets A1-A4 show the meniscus at $t=4.97,5.62,12.27$ and 13.31 , which are instants of maximum and minimum elongation in two oscillations without droplet emission. Insets B1 and B2, for $t=14.34$ and 14.46 , show the meniscus when the first droplet is emitted and when the tip recedes after the first emission burst. Insets $C 1$ and $C 2$, for $t=20.84$ and 26.83 , show the meniscus when a spindle has developed and when the spindle is about to detach. Inset $D$, for $t=26.88$, shows the meniscus at the end of the cycle, immediately after the spindle detaches and droplet emission ceases. The two black circles mark the beginning and the end of droplet emission in the cycle. 
a

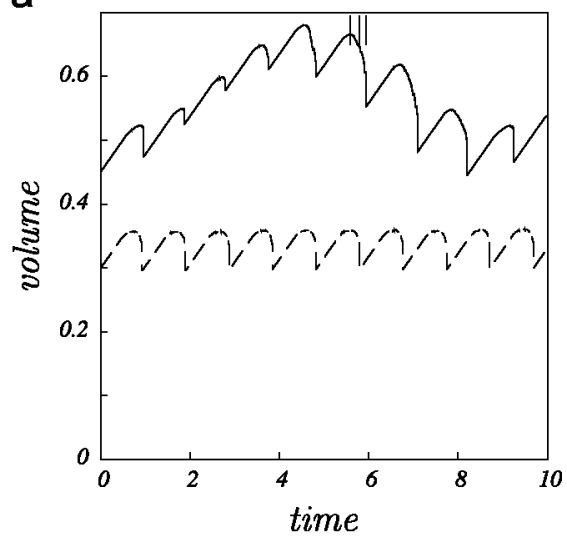

b

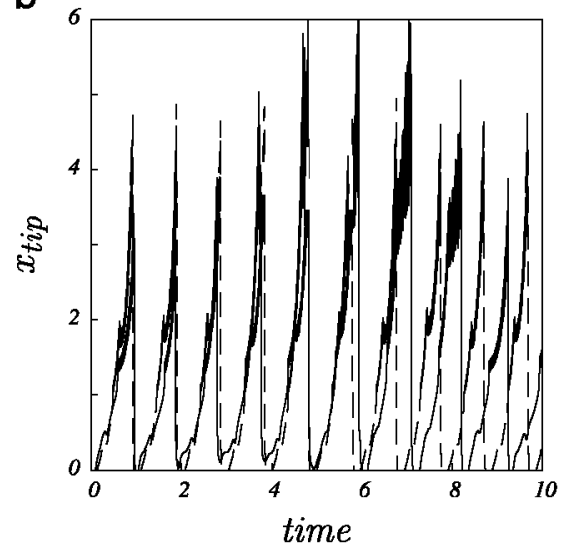

Fig. 3. Volume of the meniscus (a) and distance from the tip to the injection orifice (b) as functions of time during several cycles of the evolution for $q=0.1$ and $E_{\infty}=0.7$ (solid curves) and 0.9 (dashed curves). The three vertical tics at the top of (a) mark the instants of time at which the solution is displayed in Fig. 4.

a longer time comprising about 8 cycles (solid curve in Fig. 3(a)). This variation disappears, within the accuracy of the numerical computations, when $E_{\infty}$ is increased to 0.9 , leaving a strictly periodic regime with a period of about 0.99 ; see dashed curves in Fig. 3.

Figure 4 shows three couples of snapshots of the meniscus during a cycle, at instants of time immediately before and immediately after the emission of a droplet. The results are for $E_{\infty}=0.7$. They are keyed by vertical tics in the upper part of Fig. 3(a). The solid and dashed curves in the upper part of each panel of Fig. 4 are the electric and surface tension stresses acting on the surface of the liquid. As was advanced before, the electric stress dominates in a long region around the tip of the spindle, where strong accelerations of the liquid and emission of droplets occur. However, the surface tension stress is larger than the electric stress at the base of the spindle, which explains the formation and eventual pinchoff of a neck. In contrast to the results in Fig. 2 for $E_{\infty}=0.5$, the applied field is now sufficiently strong to restore emission after a single oscillation of the meniscus, or of a region of the meniscus around its detached tip, which leads to the continuous sequence of bursts characteristic of mode II of Juraschek and Rollgen (1998).

\section{Low frequency oscillating electric field}

We analyze now the response of the meniscus to a time periodic electric field with a square waveform. In this section we consider the limit when the period of the electric field pulses $(T)$ is large compared to the duty time $\left(t_{d}\right)$ and to the capillary time of the meniscus (of order unity in dimensionless variables). In this limit, the viscosity of the liquid, which is not expected to play an important role in the droplet emission process and has not been included in the model, will have plenty of time to damp any oscillatory motion left in the meniscus after the emission ceases, so that the following pulse of the electric field finds the meniscus essentially at rest. In addition, in a periodic regime, the time $T$ available for the flow rate to refill the meniscus with a volume equal to the volume of the emitted droplets is long compared to the duration of the emission process, so that the effect of the flow rate is small during the emission. In these conditions, the emission process can be conveniently characterized in terms of the volume of the meniscus at the beginning of the electric field pulse, $V_{i}$ say. The flow rate $q$ is set equal to zero in (7) and Eqs. (1)-(8) are solved for an initially hydrostatic meniscus of given volume. This is a spherical cap in the absence of electric field bias, and an equilibrium shape that must be precomputed when $E_{b}>0$; see, e.g. Higuera (2008). The far electric field is increased to the peak value $E_{p}$ for a time $t_{d}$, and the motion of the liquid is computed until droplet emission ceases.

\subsection{Emission modes}

Some numerical results are shown in Fig. 5 for a single electric field pulse $(T \rightarrow \infty)$, an initial dimensionless volume $V_{i}=2$, and various values of $E_{p}$ and $t_{d}$ in the absence of bias $\left(E_{b}=0\right)$. These results display two different emission modes. In the figures of the upper row, for $E_{p}=0.6$ and $t_{d}=0.8,1,1.2$ and 1.4 , the meniscus develops a tip that emits many tiny droplets while the applied field is on. This is reflected by the fast oscillations of the tip ( $\left.x_{\text {tip }}\right)$ in Fig. 5 (a), which show as blurred patches, and by the numerous small steps in the accumulated emitted volume $\left(V_{e}\right)$ in Fig. $5(\mathrm{~b})$, which give the left part of the curves the appearance of continuity. These features are the signature of this mode also in the figures that follow. When the duration of the electric field pulse, $t_{d}$, is smaller than about 1.2, the small droplets are responsible for most of the emitted volume, which increases with $t_{d}$, while for larger values of $t_{d}$ one or a few additional larger droplets are emitted after the field is turned off [large steps on the right-hand side of Fig. 5(b); see also inset in this figure]. This result reflects that, when 

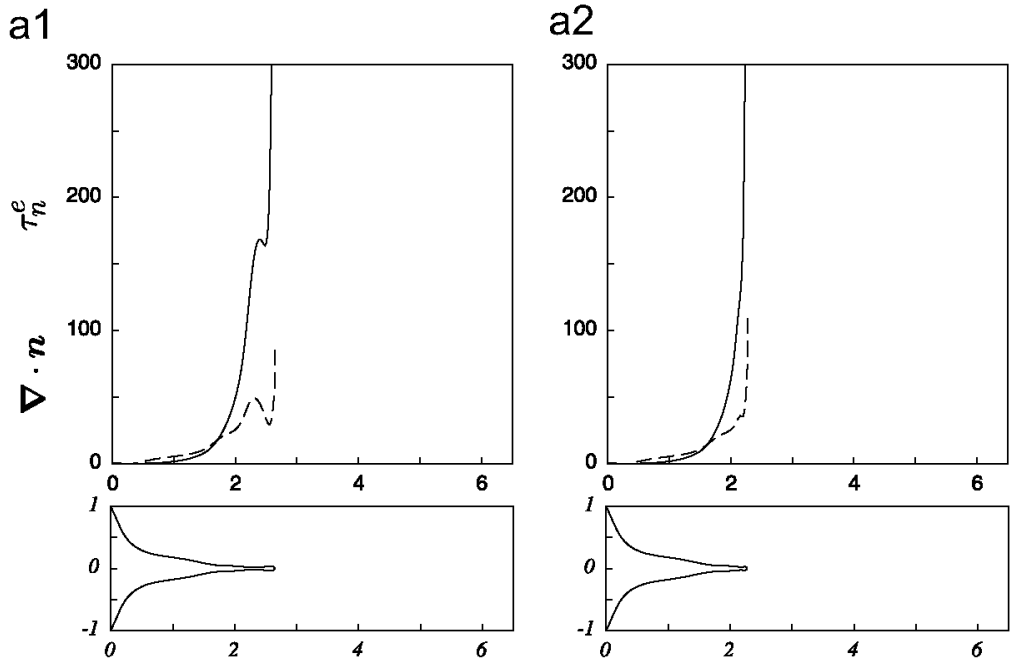

b1

b2
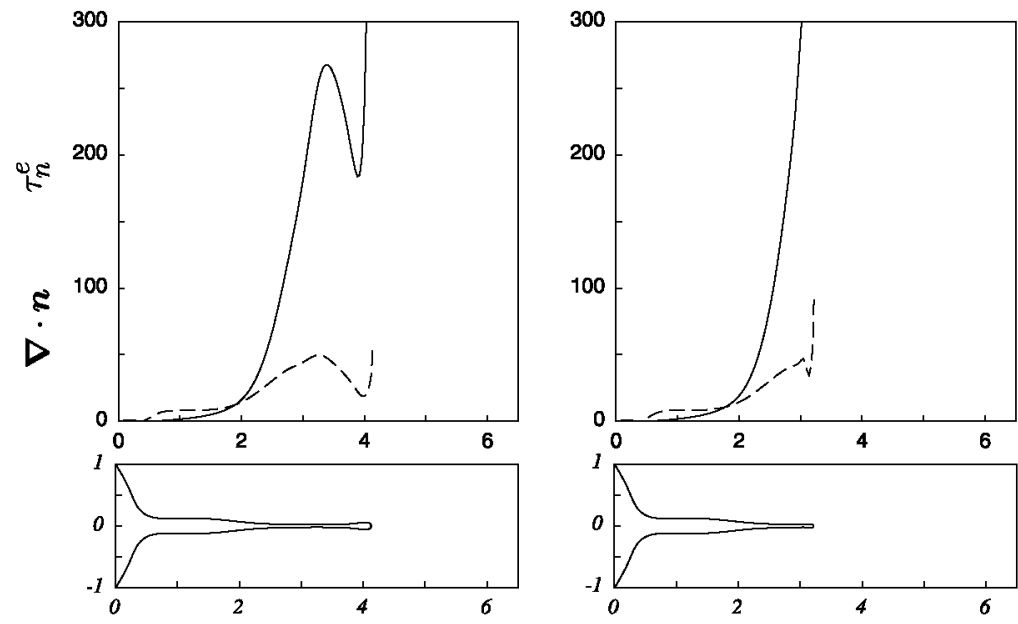

c1

c2
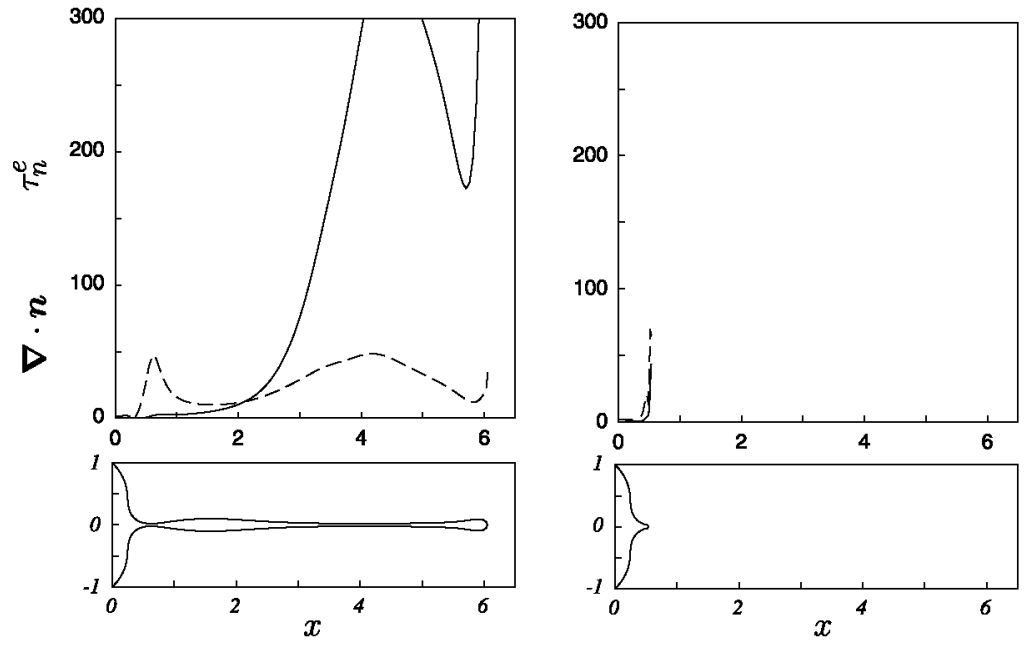

Fig. 4. Electric stress (solid curves) and surface tension stress (dashed curves) at the surface of the meniscus, as functions of the distance from the injection orifice. The shape of the meniscus is shown in the lower part of each panel. The magnitudes are shown for $q=0.1$ and $E_{\infty}=0.7$ immediately before and immediately after the droplets detachments that occur at $t=5.59$ [first row, panels (a1) and (a2)] and $t=5.78$ [second row, panels (b1) and (b2)], and immediately before and after the detachment of the spindle at $t=5.94$ [third row, panels (C1) and (C2)]. 


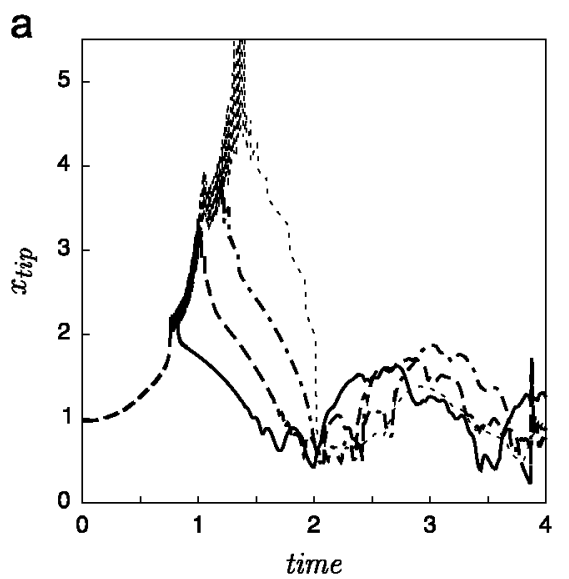

b
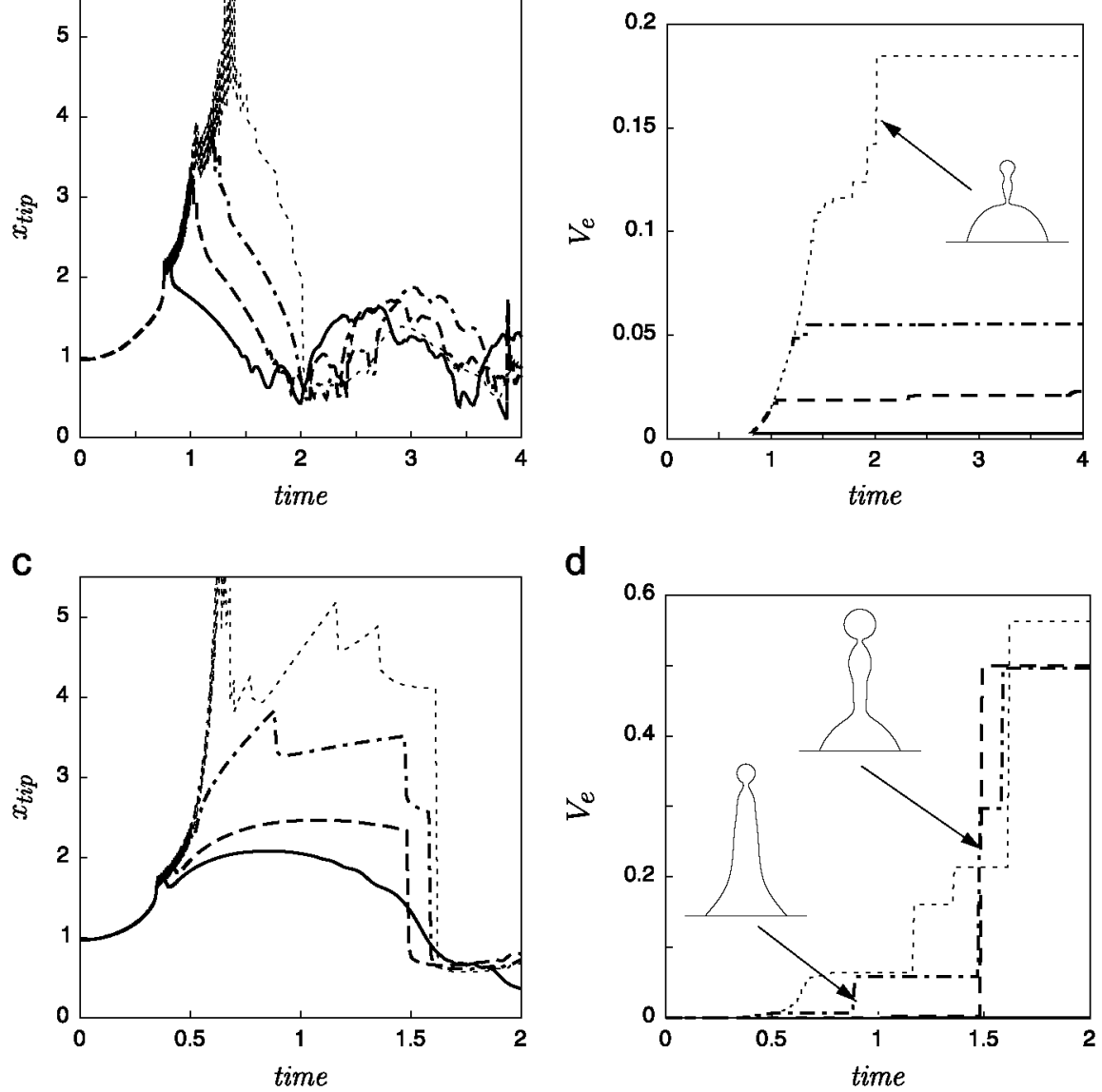

Fig. 5. Distance from the tip of the meniscus to the injection orifice $\left[x_{\text {tip }},(\mathrm{a})\right.$ and (c)] and accumulated volume of the emitted droplets $\left[V_{e},(b)\right.$ and (d)] as functions of time. In the upper row, for $E_{p}=0.6$, results are shown for $t_{d}=0.8$ (solid), 1 (dashed), 1.2 (chain), and 1.4 (dotted). In the lower row, for $E_{p}=1$, results are shown for $t_{d}=0.35$ (solid), 0.4 (dashed), 0.5 (chain), and 0.65 (dotted). Values of other parameters are $V_{i}=2$ and $E_{b}=0$. The insets show the meniscus immediately before the emission of a large droplet, after the electric field has been turned off. In (b), for $t_{d}=1.4$, a droplet of volume 0.043 is emitted at $t=2$ with initial velocity 0.55 . In $(d)$, for $t_{d}=0.5$, three droplets of volumes $0.051,0.24$ and 0.198 are successively emitted at $t=0.88,1.48$ and 1.59 , with initial velocities $2.9,1.0$ and 0.9 . Only the first and second droplets are shown.

$t_{d}$ increases, a region around the tip of the meniscus has time to gather enough momentum from the electric force acting on the liquid surface while the field is on to overcome surface tension at a later time.

Figure 5(c) and (d), for $E_{p}=1$ and $t_{d}=0.35,0.4,0.5$ and 0.65 , illustrates the second emission mode, in which most of the emitted volume is due to a few large droplets emitted after the field is turned off (the small initial emission of tiny droplets from the tip can be suppressed, at least for small values of $t_{d}$, by increasing $E_{p}$ to 1.5 ). In this case, the main effect of the electric stress is to accelerate the liquid without causing the formation of the emitting tip described in Section 3 . This seems to correspond to the microdripping mode of Kang et al. (2011) and Lee et al. (2012). The menisci in the insets of Fig. 5 may be compared to the visualization results for ethanol in Fig. 3 of Kang et al. and to Fig. 3 of Lee et al.

The second emission mode may underlie the drop-on-demand printing mechanism proposed by Mishra et al. (2010) and, to some extent, that of Kim et al. (2008), although the number, volume, velocity and direction of the emitted droplets must be controlled before an emission mechanism can be used in an application. On the other hand, a small pulse-to-pulse emission variability may be more important than strict uniformity of the droplets emitted during a pulse if all these droplets are intended to accumulate in a single spot on a substrate.

Figure 6 shows the total volume of liquid emitted as a function of the duration of the pulse, $t_{d}$, for various values of the peak electric field, $E_{p}$, and two different initial volumes of the meniscus, $V_{i}$. Also shown in this figure are results for nonzero electric bias, $E_{b}>0$, to be discussed below. In Fig. 6(a), for $V_{i}=2$, solid (red) curves are for $E_{p}=0.6,0.8,1$ and 1.5 , increasing from right to left, in the absence of bias. At the lowest value of $E_{p}$ shown, emission begins in the first mode (tiny droplets) at an onset $t_{d} \approx 0.8$ and an additional larger droplet begins to appear for $t_{d} \approx 1.2$, as was mentioned before; see Fig. 5(a) and (b). At larger values of $E_{p}$ the emission onset is smaller and large droplets appear earlier as $t_{d}$ is increased, until, for $E_{p}=1.5$, a pure second emission mode (without tiny droplets) is obtained at the onset, which is $t_{d} \approx 0.19$ for this value of $E_{p}$. The dotted segment of the leftmost solid curve of Fig. 6(a) marks the abrupt transition that occurs in this case from oscillation without droplet emission to emission of 

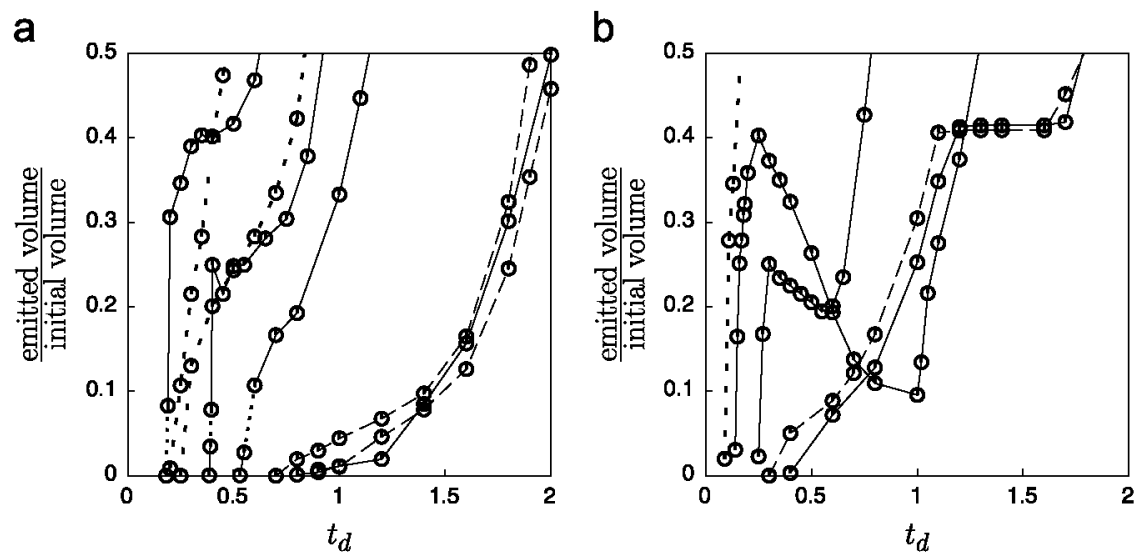

Fig. 6. Total volume of emitted droplets scaled with the initial volume of the meniscus as a function of the duration of the electric field pulse, $t_{d}$. In (a), for $V_{i}=2$, solid curves are for $E_{p}=0.6,0.8,1$ and 1.5 , increasing from right to left, with $E_{b}=0$; dashed curves are for $E_{p}=0.6$ with $E_{b}=0.3$ and 0.4 , and dotted curves are for $E_{\mathrm{p}}=1$ with $E_{b}=0.3$ and 0.4 , increasing from right to left in both cases. In (b), for $V_{i}=1$, solid curves are for $E_{p}=1,1.5$ and 2 , increasing from right to left in the lower part of the figure, with $E_{b}=0$; the dashed curve is for $E_{p}=1, E_{b}=0.5$; and the dotted curve is for $E_{p}=2$, $E_{b}=0.5$. (For interpretation of the references to color in this figure caption, the reader is referred to the web version of this article.)

a single, fairly large droplet. Similar abrupt transitions occur for $E_{p}=1$ (at $t_{d} \approx 0.38$ ) and $E_{p}=0.8$ (at $t_{d} \approx 0.55$ ), though in these cases small droplets are emitted in the first mode below the transition point. The second emission mode dominates for large values of $t_{d}$ at the three largest $E_{p}$ in Fig. 6(a). However, the droplets tend to be large in these conditions; see Fig. 5(c) and (d). The number of large droplets emitted and their volumes and instants of emission depend on $t_{d}$ and $E_{p}$, but this dependence is not smooth, reflecting a complex dynamics of the meniscus under the abrupt changes of the electric stress that occur when the electric field is turned on and off, and the abrupt changes of the surface tension stress that occur when droplets detach. This complexity is also reflected by the evolution of $x_{t i p}$ in Figs. 5 and 8 after droplet emission ceases.

\subsection{Effect of an electric field bias}

The dashed (blue) and dotted (green) curves in Fig. 6 show the effect of an electric field bias. Consider Fig. 6(a). When $E_{p}=0.6$, a moderate bias ( $E_{b}=0.3$, rightmost dashed curve) does not qualitatively change the first emission mode, but significant changes occur when the bias is increased to $E_{b}=0.4$. These changes are not clearly reflected in Fig. 6(a) but can be appreciated in Fig. 7(a) and (b), which show results for the same parameter values as Fig. 5(a) and (b) except for the presence of the bias. The increased bias affects the evolution of the meniscus during the electric field duty time (through the initial elongation it induces) and beyond. It leads to the emission of large droplets at smaller values of $t_{d}$ than in the absence of bias (compare the steps and final values of $V_{e}$ in Figs. 7(b) and 5(b)), and makes their emission more ordered (aligned steps in Fig. 7(b) and (d)).

The two dotted (green) curves in Fig. 6(a), for $E_{p}=1$ and $E_{b}=0.3$ and 0.4 , show that the bias smooths the abrupt transition at the beginning of the second emission mode, and thus enlarges the narrow range of $t_{d}$ where relatively small droplets can be obtained in this mode when $E_{b}=0$. The steps in Fig. 7(d) show the emission of one (in the two lower curves) or two (in the upper curves) droplets in a range of $t_{d}$ where very little emission occurs in the absence of bias.

In all the cases, the bias has the undesirable effect of increasing the size of the droplets for large values of $t_{d}$. In addition, the bias cannot be increased much above the values in Fig. 6. Larger biases lead to sharp-tipped menisci that do not recede and keep emitting tiny droplets after the peak electric field is turned off, even though the bias alone would not cause emission from an initially round meniscus. This behavior can already be seen in Fig. 7. The emitting tip (blurred area) persists up to about $t=2$ in Fig. 7(a) and $t=1.8$ in Fig. 7(c), which are larger than the pulse duration $\left(t_{d}\right)$ and independent of it. It is thus the bias, rather than $E_{p}$ or $t_{d}$, that determines the lifetime of the tip, whose detachment leads to the first large droplet registered in Fig. 7(b) and (d). In some cases the bias is enough to stretch the stump left at detachment and rekindle emission, which happens at about $t=3.5$ in Fig. 7(a) and $t=2.8$ in Fig. 7(c). This behavior is a consequence of the selfintensification of the electric field around a pointed equipotential surface. It is related to the hysteretical response whereby the maximum electric field at which a meniscus of given volume can be in equilibrium, and above which emission of droplets begins, is larger than the minimum electric field at which emission is quenched and the tip of the meniscus recedes (see Cloupeau \& Prunet-Foch, 1989; Higuera, 2010).

\subsection{Effect of the volume of the meniscus}

Figure 6(b) shows results for an initial volume $V_{i}=1$ and $E_{p}=1,1.5$ and 2, while Fig. 8 shows the time evolution of the tip of the meniscus and the accumulated volume of emitted droplets for a few sample cases. Only the main differences with the 
a

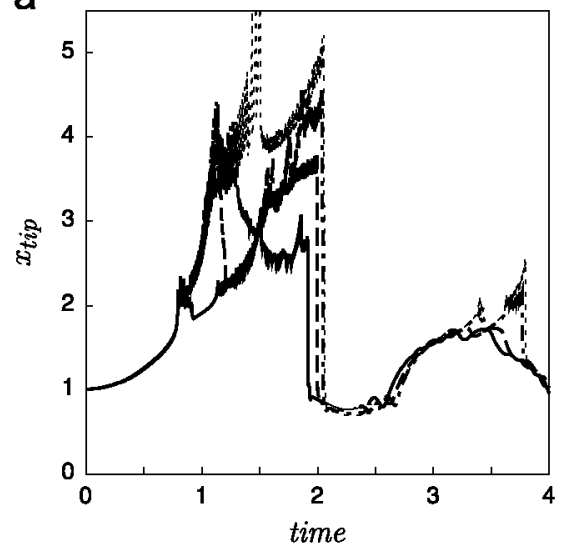

C

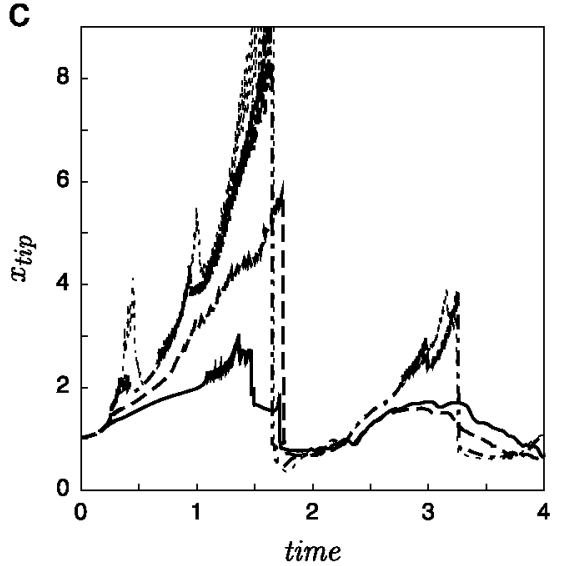

b

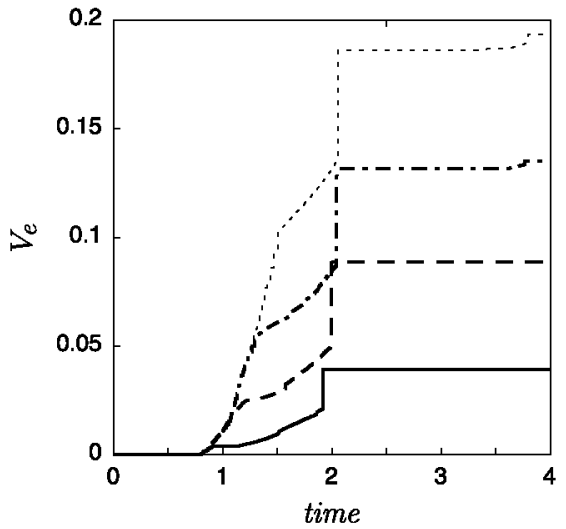

d

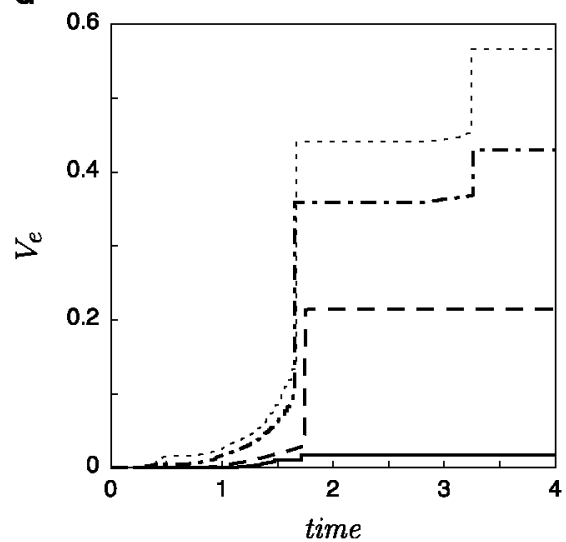

Fig. 7. Distance from the tip of the meniscus to the injection orifice $\left[x_{\text {tip }},(a)\right.$ and $\left.(c)\right]$ and accumulated volume of the emitted droplets [ $V_{e}$, (b) and (d)] as functions of time. In the upper row, for $E_{p}=0.6$, results are shown for $t_{d}=0.8$ (solid), 1 (dashed), 1.2 (chain), and 1.4 (dotted). In the lower row, for $E_{p}=1$, results are shown for $t_{d}=0.2$ (solid), 0.25 (dashed), 0.3 (chain), and 0.35 (dotted). Values of other parameters are $V_{i}=2$ and $E_{b}=0.4$.

results for $V_{i}=2$ will be discussed. The results for $E_{p}=1$ and $E_{b}=0$ (rightmost solid curve in the lower part of Fig. 6(b)) show a continuous increase in the emitted volume with the duration of the electric field pulse from the onset of emission to $t_{d} \approx 1.2$, followed by a plateau of constant emitted volume that extends to $t_{d} \approx 1.65$, before the emitted volume increases again. This plateau reflects the fact that the first naturally occurring emission pulse under a DC field $E_{\infty}=1$ ends at a time $t \approx 1.18$ after the DC field is applied. Due to the lapse of no emission, the curves in Fig. $8(\mathrm{~b})$ for $t_{d}=1.4$ (chain, red), in the plateau of Fig. $6(\mathrm{~b})$, and $t_{d}=1.8$ (dotted, black), at the right of the plateau, coincide for $t$ smaller than about 1.6 , at which moment emission resumes in the second case.

The dashed curve in Fig. 6(b) and Figs. 8(c) and (d) show the effect of an electric field bias. As for $V_{i}=2$, the bias decreases the volume of the large droplets and the minimum value of $t_{d}$ at which these droplets appear. However, the bias does not suppress the emission of tiny droplets or the plateau discussed above.

The results for $E_{p}=1.5$ and 2 in Fig. 6(b) (solid curves at the left-hand side of the figure) display a range of pulse duration, $t_{d}$, where the emitted volume decreases when $t_{d}$ increases. Inspection of the numerical solutions shows that, in this range, the increase of momentum due to the electric stress concentrates around the tip of the meniscus, leading to the emission of many small droplets with high velocity, but the momentum imparted to the rest of the meniscus decreases with increasing $t_{d}$, and so do the velocity with which the meniscus elongates after the end of the electric field pulse and the emission of droplets in this stage. When $E_{p}=2$, utilization of electric stresses to generate small droplets is maximum for $t_{d} \approx 1$, at which point emission of large droplets after the end of the pulse is nearly null and the total emitted volume is minimum at about one tenth of the initial volume of the meniscus. Emission of large droplets rapidly regains importance when $t_{d}$ is further increased; see dotted curve in Fig. 8(f). This figure also shows that, due to the non-monotonic response, the accumulated volume of emitted droplets is nearly the same for $t_{d}=0.3$ (solid curve) and for $t_{d}=1.2$ (dotted curve), though most of this volume is emitted as a single droplet of volume 0.358 in the first case and as a spray of tiny droplets followed by a larger droplet of volume 0.138 in the second case. 
a
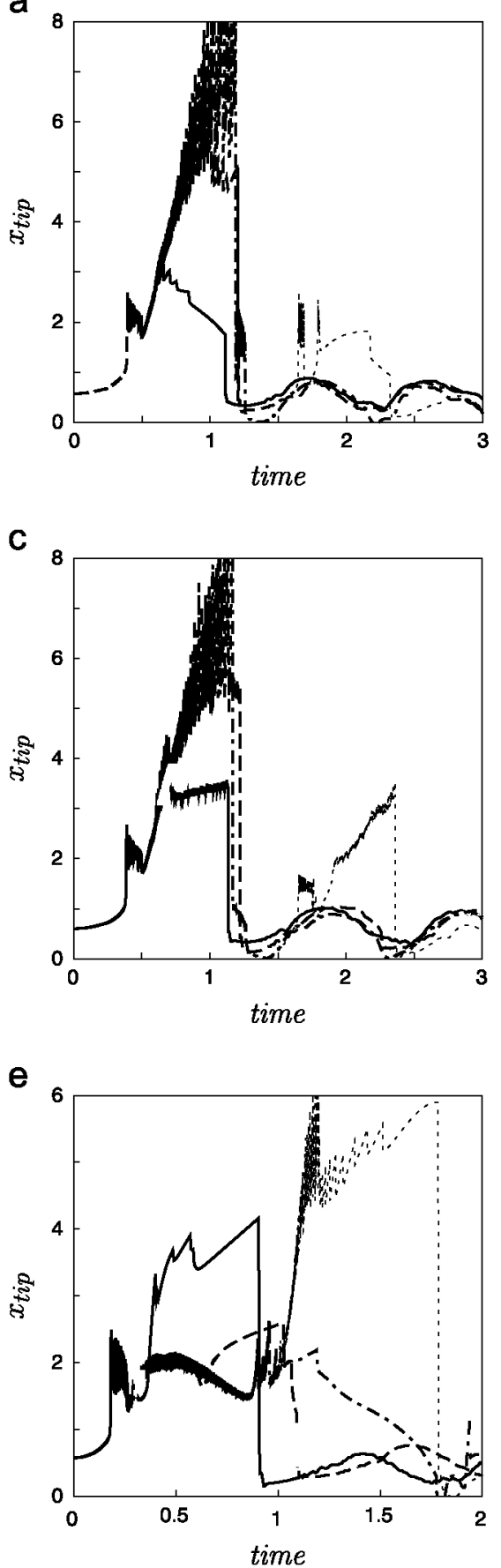

b

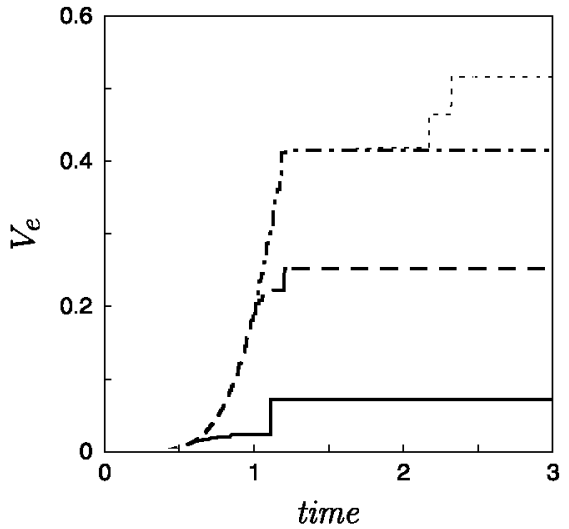

d

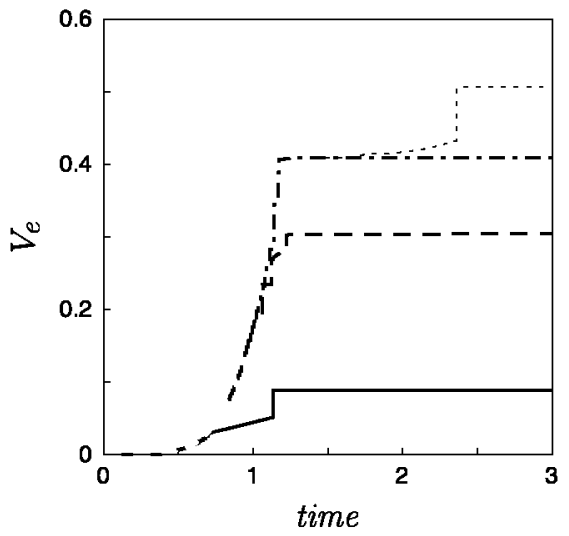

f

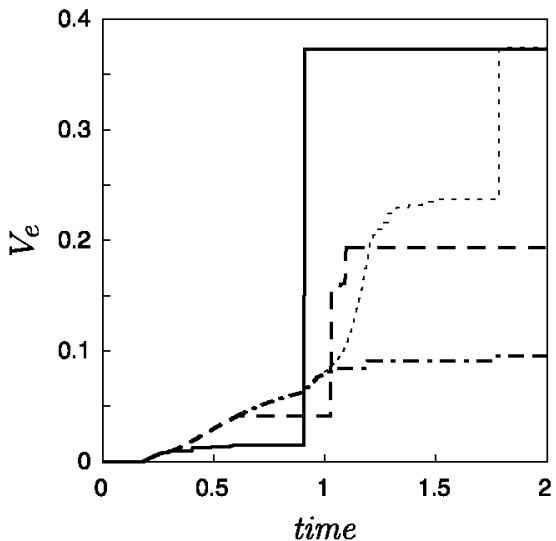

Fig. 8. Distance from the tip of the meniscus to the injection orifice $\left[x_{t i p},(a),(c)\right.$ and (e)] and accumulated volume of the emitted droplets $\left[V_{e}\right.$, (b), (d) and (f)] as functions of time for $V_{i}=1$. In the upper row, for $E_{p}=1$ and $E_{b}=0$, results are shown for $t_{d}=0.6$ (solid), 1 (dashed), 1.4 (chain), and 1.8 (dotted). In the middle row, for $E_{p}=1$ and $E_{b}=0.5$, results are shown for the same values of $t_{d}$. In the lower row, for $E_{p}=2$ and $E_{b}=0$, results are shown for $t_{d}=0.3$ (solid), 0.6 (dashed), 1 (chain), and 1.2 (dotted). (For interpretation of the references to color in this figure caption, the reader is referred to the web version of this article.)

\subsection{Additional comments}

The complex response of the meniscus to a pulsed electric field revealed by the results of this section suggest that electrohydrodynamic generation of droplets for printing applications requires careful tuning of the control parameters. The volume of liquid emitted per cycle increases with the duration of the electric field pulse, except in the regions of negative 
slope and the plateau of Fig. 6(b), but the fraction of this volume emitted as very small droplets also changes with the duration of the pulse and the peak field. An appropriate electric field bias may decrease the size of the large droplets and favor and regularize their emission, but an excessive bias may increase the droplet size and render the emission uncontrollable.

The periodic response of the system to a periodic pulsed electric field with a frequency small compared to the capillary frequency of the meniscus can be obtained by drawing curves similar to those of Fig. 6(a) and (b) in a single diagram. For a given pulse frequency, the emitted volume shown in such diagram would be proportional to the flow rate, and the volume of the meniscus for given values of $E_{p}, E_{b}$ and $t_{d}$, as well as the emission mode, could be determined by interpolating the results computed for different values of $V_{i}$. The fact that the emitted volume increases with $V_{i}$ at constant $E_{p}$ (compare curves for the same $E_{p}$ in Fig. 6(a) and (b)) shows that the volume of the meniscus would increase with the flow rate.

In the experiments of Kang et al. (2011) and Lee et al. (2012), the volume emitted per cycle of the applied voltage is changed by changing the voltage frequency at constant flow rate and peak voltage. Figure 5 of Kang et al. and Fig. $5 \mathrm{~b}$ of Lee et al. show that the volume emitted per cycle decreases when the voltage frequency is increased, as required by mass conservation. The images in the upper row of Fig. 4 of Kang et al. and in Fig. 5(a) of Lee et al. show that the volume of the meniscus, $V_{i}$, also decreases when the voltage frequency is increased. Therefore the emitted volume increases with $V_{i}$, which supports the numerical results above. (Notice that the meniscus volume $V_{i}$ is the volume before droplet emission, which should include the volume of the droplet visible in the images of Kang et al. and Lee at al.)

\section{Approximate analysis for high frequency oscillating electric field}

Analysis of the systems proposed to achieve high speed droplet emission with electric fields pulsed at frequencies of the order of the capillary frequency of the meniscus is complicated, due not only to the response of the meniscus but also to the complex interaction of the meniscus and the liquid feeding system. Here, to avoid this complexity and focus on the dynamics of the meniscus, the drastic simplifying assumption is made that the volume of the meniscus is a constant during its whole evolution. While this cannot be strictly true for a meniscus that emits droplets, the assumption is still expected to capture some elements of the liquid dynamics for reasonable feeding systems when the volume of liquid emitted per cycle of the electric field, or during the response time of the feeding system, is small compared to the volume of the meniscus.

The solution of the model problem embodying the assumption above depends on the dimensionless parameters $E_{p}, E_{b}, t_{d}$ and $T$ defined in Section 2, and on the dimensionless volume of the meniscus, $V_{m}$ (scaled with the cube of the radius of the injection orifice). The flow rate for a time periodic solution of this problem is $q=V_{e} / T$, where $V_{e}$ is the accumulated volume of the droplets emitted in a cycle of the electric field, which is to be found as part of the solution. In the numerical treatment, the condition of constant volume is imposed by immediately reinjecting into the meniscus the volume of any emitted droplet. A hydrostatic meniscus of volume $V_{m}$ is assumed as an initial condition.

Numerical solutions of the simplified problem for $V_{m}=2$ show that the emission of droplets is not periodic for $E_{p}=0.6$ at any value of the period $T$ tested (below 16 ). This $E_{p}$ is the smallest peak electric field discussed in the previous section, for which emission of small droplets was obtained with a single pulse. Some results for $E_{p}=1$ are summarized in Fig. 9 . As can be seen, periodic emission is obtained for a pulse duration $t_{d}=0.4$ (dashed curves) when $T=8$, for which the volume emitted per pulse is $V_{e} / T \approx 0.07$, while the solution is not far from periodic when $T=4$ or 2 , with $V_{e} / T \approx 0.072$ and 0.23 , respectively. Emission occurs mainly in the second mode (large droplets) in the three cases. Inspection of the numerical solution for $T=8$ shows that the electric stresses acting on the meniscus lead to the formation of a tip that begins emitting tiny droplets while, at the same time, a larger region of the meniscus gathers momentum and elongates. Emission from the tip ceases when the electric field is turned off, but the larger region keeps elongating and leads to a filament that breaks into two or three larger droplets before the meniscus recedes. The meniscus then undergoes four capillary oscillations before the

a

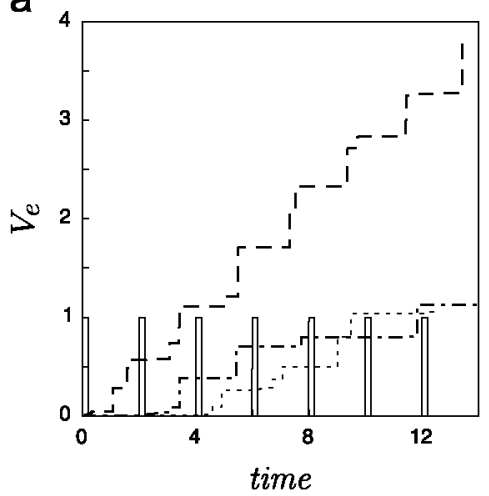

b

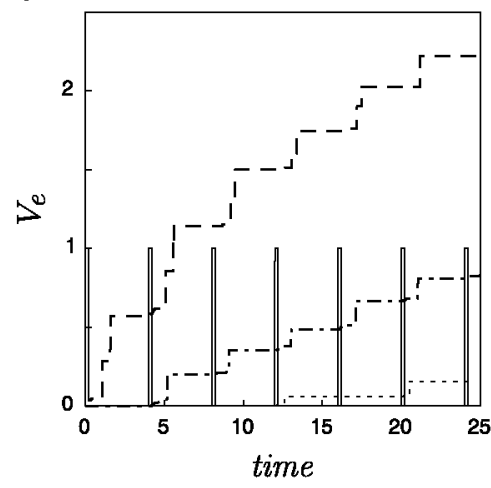

C

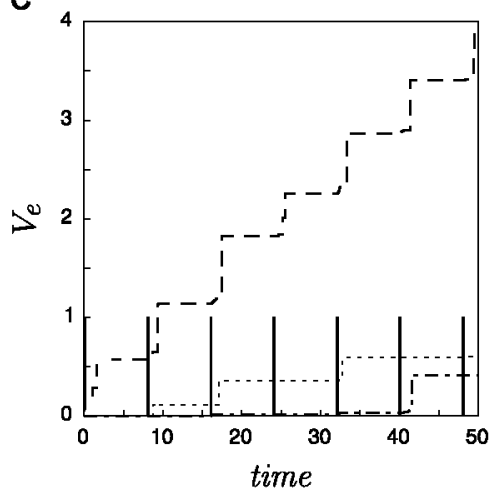

Fig. 9. Accumulated volume of emitted droplets as a function of time for $V_{m}=2, E_{p}=1, T=2(\mathrm{a}), 4(\mathrm{~b})$ and $8(\mathrm{c})$, and $\left(t_{d}, E_{b}\right)=(0.4,0)$ (dashed curves), $(0.2,0)$ (dotted curves), and $(0.2,0.3)$ (chain curves). The vertical solid lines mark the electric field pulses. 
electric field is turned on again and the cycle repeats. The results for $T=4$ are similar, except that now the meniscus has no time to complete two capillary oscillations before the electric field is turned on again. Therefore the following pulse does not find the meniscus at its maximum elongation, which increases the time required to form a new emitting tip. When $T=2$, the emission elicited by a pulse ends shortly before the electric field is turned on again. The meniscus is rather blunt at this instant of time and cannot develop an emitting tip before the end of the pulse, so that emission in the first mode (tiny droplets) does not occur.

The solution ceases to be periodic when the duration of the pulses is reduced to $t_{d}=0.2$ (dotted curves). Then, as noted by Kang et al. (2011) and Lee et al. (2012), the flow does not follow the electric field, and emission fails in some cycles. The electric stresses due to individual electric field pulses still lead to a droplet-emitting tip in some of the pulses, but emission of large droplets in the second mode requires the cumulative effect of several pulses on the oscillating meniscus, and it is not periodic.

Periodic emission can be restored for $t_{d}=0.2$ and $T=4$ by adding a bias $E_{b}=0.3$ (chain curves), which leads to relatively small droplets with $V_{e} / T \approx 0.037$. In this case the tip of the meniscus keeps emitting tiny droplets for some time after the peak electric field is turned off. This is followed by the emission of a large droplet and by a full oscillation of the meniscus without further emission, in such a way that the next electric field pulse finds the meniscus at nearly its maximum elongation and can easily form a new emitting tip. The bias decreases the period of oscillation of the meniscus by changing its shape and decreasing the volume of liquid that oscillates. The same bias $\left(E_{b}=0.3\right)$ cannot make the solution periodic for $T=8$ or $T=2$, apparently because the oscillation of the meniscus fails to synchronize with the pulsed electric field.

To further examine the effect of the bias, $E_{b}$ has been changed keeping $E_{p}=1$ and $t_{d}=0.2$. The results are summarized in Fig. 10. Periodic emission has never been obtained for $T=2$, at least during the seven first pulses of the electric field shown in Fig. $10(a)$, although the solution for $E_{b}=0.4$ tends to become periodic after about 10 pulses, with $V_{e} / T \approx 0.25$ and noticeable emission in both modes.

Figure $10(\mathrm{~b})$ and (c) shows that periodic emission is obtained in narrow ranges of $E_{b}$ for $T=4$ and 8 . For $T=4$, a $E_{b}=0.2$ does not change much the solution without bias; a $E_{b}=0.3$ leads to the periodic solution discussed above; and a $E_{b}=0.4$ spoils this periodicity. In the latter case the meniscus sometimes keeps emitting for a considerable time after the peak field is turned off, and becomes irresponsive to the following few pulses. Thus, the dotted curve in Fig. 10(b) shows two emission bursts after pulses 2 and 5, and no emission after pulses 3 and 4 . Inspection of the solution shows that this relatively large bias is able to restore emission in the first mode (tiny droplets) after an emission burst, provided it has not left a very blunt meniscus. This makes the dynamics less dependent on the electric field pulses, which may find the meniscus in different phases of its evolution and cannot enforce periodicity.

Periodic emission for $T=8$ is obtained when $E_{b}=0.4(\operatorname{Fir} 1 \cap(\mathrm{c}))$. The evolution of the meniscus is similar to that described above for $T=4$ and $E_{b}=0.3$, except that the number of oscillations following the emission of a large drop is now larger.

In all the cases, the results show that a condition for periodic emission is that the capillary oscillations of the meniscus be able to lock on to the pulsed electric field. The bias affects this synchronization by changing the frequency of the meniscus. A large bias induces continuous emission of tiny droplets, which decreases the sensitivity of the meniscus to the peaks of the applied field.

Results for $E_{p}=1.5$ (not displayed) are qualitatively similar to those for $E_{p}=1$, though the droplets generated are somewhat larger. For example, $V_{e} / T \approx 0.118$ for $t_{d}=0.2$ and $T=4$ with $E_{b}=0.3$. The increase of $E_{p}$ allows to decrease $t_{d}$. Thus, periodic emission with $V_{e} / T \approx 0.087$ is obtained in the absence of bias for $t_{d}=0.2$ when $T=8$, though not for smaller values of $T$.

Similar computations have been carried out for $V_{m}=1$. The minimum peak electric field for which periodic emission is obtained is now $E_{p}=1.5$, for which $V_{e} / T \approx 0.061$ when $t_{d}=0.3$ and $T=4$, a value that decreases to $V_{e} / T \approx 0.036$ when a bias $E_{b}$ is added.

In summary, the limited results obtained for the model problem with constant meniscus volume suggest that periodic emission of droplets can be achieved in some cases for dimensionless electric field pulse frequencies (scaled with $\gamma^{1 / 2} / \rho^{1 / 2} a^{3 / 2}$ ) up to about 0.25 (chain curve in Fig. $10(\mathrm{~b})$ ) and even up to 0.5 if a long initial transient spanning about ten

a

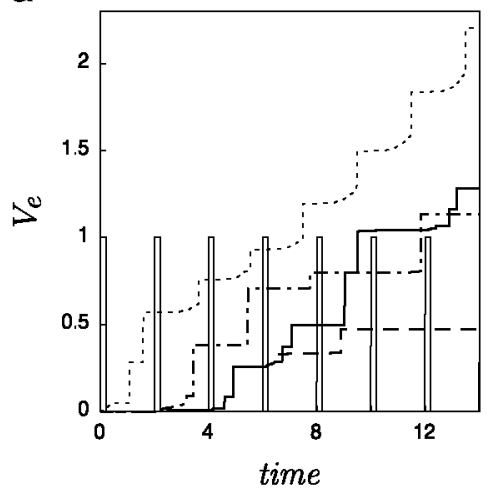

b

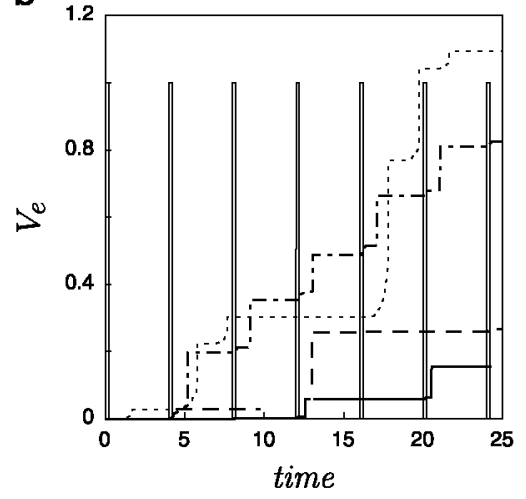

C

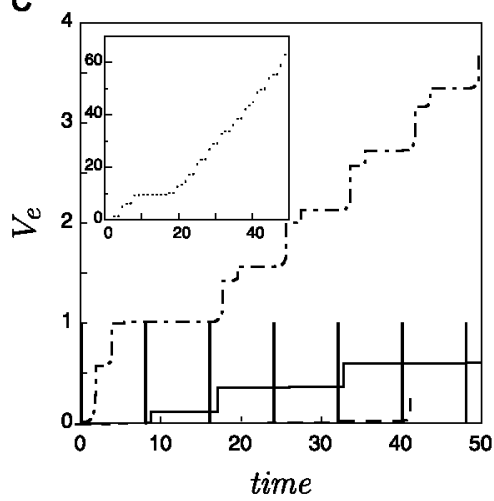

Fig. 10. Accumulated volume of emitted droplets as a function of time for $V_{m}=2, E_{p}=1, t_{d}=0.2$, and various values of $E_{b}$. In (a) and (b), for $T=2$ and 4 , respectively, $E_{b}=0$ (solid), 0.2 (dashed), 0.3 (chain) and 0.4 (dotted). In (c), for $T=8, E_{b}=0$ (solid), 0.3 (dashed) and 0.4 (chain). The inset shows results for $E_{b}=0.5$ (dotted), which do not fit in the vertical scale of the figure. The vertical solid lines mark the electric field pulses. 
pulses of the electric field is allowed. Increasing the peak electric field and the duration of the pulse (from $E_{p}=0.6$ to 1 and from $t_{d}=0.2$ to 0.4 in the cases discussed above) tends to regularize the emission, but at the expense of increasing the size of the droplets. An electric field bias may regularize the emission and decrease the size of the droplets, but this happens only in a narrow range of bias for given values of other parameters (cf. Fig. 10). The results suggest that simple rules stated sometimes, according to which the duration of the electric field pulse determines the droplet volume, the time interval between pulses controls the droplet deposition rate, and the electric field bias decreases the size of the droplets and increases the maximum attainable frequency, may not fully account for the complex dynamics of electrified menisci.

\section{Conclusions}

The emission of droplets from a meniscus of an inviscid liquid of infinite electrical conductivity subject to an electric field has been studied numerically. Two processes with very different time scales may coexist in the evolution of the meniscus. The fast process is the formation and stretching of a filament at the tip of the meniscus, under the action of intense electric stresses due to the self-intensified electric field around the filament, which end up producing the emission of tiny droplets from the end of the filament. The slow process is an oscillation of the meniscus with a characteristic time of the order of the capillary time.

Under a DC electric field, the pulsating emission of droplets occurs in bursts, each burst comprising the formation of an elongated tip, the emission of a number of tiny droplets, and the detachment of the emitting tip as one or two larger droplets. At low electric fields these bursts group in sequences interspersed with oscillations of the meniscus without droplet emission, while at higher electric fields the bursts become time periodic. These results are consistent with axial modes I and II of Juraschek \& Rollgen (1998). The detachment of the emitting tip may be the means by which the imbalance between mass emission and mass supply to the meniscus hypothesized by these authors is brought about.

Two distinct emission modes, or a combination of the two, are possible under a pulsed electric field. The electric stresses that act on the surface of the liquid for a limited time may lead to the formation of a tip that emits very small droplets, as with a DC field but only for the duration of the pulse, or they may accelerate a larger volume of liquid and cause the emission of one or a few large droplets after the electric field is turned off. The total volume of liquid emitted per pulse and the dominant emission mode have been computed as functions of pulse duration and field intensity and bias for cases when the frequency of the pulsed field is small compared to the capillary frequency of the meniscus. The results show that short pulses of intense field favor the generation of post-pulse droplets, and that an appropriate bias may decrease the size of these droplets and enlarge the range of pulse duration where their emission occurs. However, the response of the electrified meniscus is very sensitive to the control parameters, and desirable emission characteristics are obtained only in small regions of the parameter space.

Droplet emission due to an electric field pulsed with a frequency of the order of the capillary frequency has been analyzed in the framework of a simplified constant volume meniscus model. In addition to the sensitivity to control parameters already pointed out for low frequency fields, the numerical results show that periodic emission at high frequency depends on synchronizing the pulsed electric force due to the applied field with the capillary oscillations of the meniscus, whose frequency in turn depends on the bias and peak electric field. The average emission rate and the frequency of the electric field above which droplet emission fails to follow the field have been examined.

The main results of the work can be summarized as follows: (i) The dynamics of the meniscus and the emitting tip in the two pulsating regimes that appear under a DC voltage have been described. The formation of a neck and the subsequent pinchoff of the emitting tip are shown to cause the end of droplet emission bursts. (ii) Two different emission modes have been found and characterized for low frequency pulsed voltages. The effects of the voltage amplitude, duty time and bias on the emission mode and the volume of emitted droplets are described. The numerical results qualitatively capture features observed in experiments, such as the shape of the meniscus and the variation of its volume with flow rate or with the volume of liquid emitted per pulse of the applied voltage. (iii) The evolution of an oscillatory meniscus forced by a voltage pulsed at a frequency of the order of the capillary frequency of the meniscus has been described. The conditions under which pulsating emission of droplets fails to occur at the frequency of the applied voltage have been ascertained. The roles of voltage frequency and bias have been investigated.

\section{Acknowledgments}

This work was supported through projects DPI2010-20450-C03 and CSD2010-00010 (Ministerio de Ciencia e Innovación), and S2009/ENE-1597 (Comunidad de Madrid).

\section{References}

Alexander, M.S., Paine, M.D., \& Stark, J.P.W. (2006). Pulsation modes and the effect of applied voltage on current and flow rate in nanoelectrospray. Analytical Chemistry, 78, 2658-2664.

Basaran, O.A., Gao, H., \& Bhat, P.P. (2013). Nonstandard injets. Annual Review of Fluid Mechanics, 45, 85-113.

Batchelor, G.K. (1967). An introduction to fluid dynamics. Cambridge University Press: Cambridge. 
Chen, A.U., \& Basaran, O.A. (2002). A new method for significantly reducing drop radius without reducing nozzle radius in drop-on-demand drop production. Physics of Fluids, 14, L1-L4.

Chen, C.-H., Saville, D.A., \& Aksay, I.A. (2006). Scaling laws for pulsed electrohydrodynamic drop formation. Applied Physics Letters, $89,124103$.

Cheng, K., Yang, M.H., Chiu, W.W., Huang, C.Y., Chang, J., \& Ying, T.F., et al. (2005). Ink-jet printing self-assembled polyelectrolytes and electroless plating: Low cost fabrication of circuits on a flexible substrate at room temperature. Micromolecular Rapid Communications, 26, 247-264.

Choi, H.K., Park, J.-U., Park, O.O., Ferreira, P.M., Georgiadis, J.G., \& Rogers, J.A. (2008). Scaling laws for jet pulsations associated with high-resolution electrohydrodynamic printing. Applied Physics Letters, 92, 123109.

Cloupeau, M., \& Prunet-Foch, B. (1989). Electrostatic spraying of liquids in cone-jet mode. Journal of Electrostatics, 22, $135-159$.

Cloupeau, M., \& Prunet-Foch, B. (1994). Electrohydrodynamic spraying functioning modes: A critical review. Journal of Aerosol Science, 25, 1021-1036.

Fernández de la Mora, J. (2007). The fluid dynamics of Taylor cones. Annual Review of Fluid Mechanics, 39, 217-243.

Fernández de la Mora, J., \& Loscertales, I.G. (1994). The current emitted by highly conducting Taylor cones. Journal of Fluid Mechanics, 260, 155-184.

Hebner, T.R., Wu, C.C., Marcy, D., Lu, M.H., \& Sturm, J.C. (1998). Ink-jet printing of doped polymers for organic light emitting devices. Applied Physics Letters, $72,519-521$.

Higuera, F.J. (2004). Liquid flow induced by ion evaporation in an electrified meniscus. Physical Review E, 69, 066301.

Higuera, F.J. (2007). Emission of drops from the tip of an electrified jet of an inviscid liquid of infinite electrical conductivity. Physics of Fluids, 19, 072113.

Higuera, F.J. (2008). Model of the meniscus of an ionic-liquid ion source. Physical Review E, 77, 026308.

Higuera, F.J (2010). Numerical computation of the domain of operation of an electrospray of a very viscous liquid. Joumal of Fluid Mechanics, 648, 35-52.

Hijano, A.J., Loscertales, I.G., Ibáñez, S.E., \& Higuera, F.J. (2012). Periodic emission of droplets from an electrified meniscus. In Bull. Am. Phys. Soc. 65th Annual meeting of the division of fluid dynamics (pp. 307-308). ISSN: 0003-0503

Hinch, E.J. (1991). Perturbation methods. Cambridge University Press: Cambridge.

Jayasinghe, S.N., Edirisinghe, M.J., \& Wilde, T.D. (2002). A novel ceramic printing technique based on electrostatic atomization of a suspension. Materials Research Innovations, 6, 92-95.

Jayasinghe, S.N., \& Townsend-Nicholson, A. (2006). Stable electric-field driven cone-jetting of concentrated biosuspensions. Lab on a Chip, 6, 1086-1090.

Jaworek, A., \& Krupa, A. (1999). Classification of the modes of EHD spraying. Journal of Aerosol Science, 30, 873-893.

Jaworek, A., \& Sobczyk, A.T. (2008). Electrospraying route to nanotechnology: An overview. Journal of Electrostatics, 66, 197-219.

Juraschek, R., \& Rollgen, F.W. (1998). Pulsation phenomena during electrospray ionization. International Journal of Mass Spectrometry, $177,1-15$.

Kang, D.K., Lee, M.W., Kim, H.Y., James, S.C., \& Yoon, S.S. (2011). Electrohydrodynamic pulsed-inkjet characteristics of various inks containing aluminum particles. Joumal of Aerosol Science, 42, 621-630.

Kim, J., Oh, H., \& Kim, S.S. (2008). Electrohydrodynamic drop-on-demand patterning in pulsed cone-jet mode at various frequencies. Journal of Aerosol Science, 39, 819-825.

Landau, L.D., \& Lifshitz, E.M. (1960). Electrodynamics of contimuous media. Pergamon Press: Oxford.

Lee, M.W., Kang, D.K., Kim, N.Y., Kim, H.Y., James, S.C., \& Yoon, S.S. (2012). A study of ejection modes for pulsed-DC electrohydrodynamic inkjet printing. Journal of Aerosol Science, 46, 1-6.

Lee, D.Y., Shin, Y.S., Park, S.E., Yu, T.U., \& Hwang, J. (2007). Electrohydrodynamic printing of silver nanoparticles by using a focused nanocolloid jet. Applied Physics Letters, 90, 081905.

Marginean, I., Nemes, P., Parvin, L., \& Vertes, A. (2006). How much charge is there on a pulsating Taylor cone?. Applies Physics Letters, $89,064104$.

Marginean, l., Nemes, P., \& Vertes, A. (2006). Order-chaos-order transitions in electrosprays: The electrified dripping faucet. Physical Review Letters, 97, 064502

Marginean, I., Nemes, P., \& Vertes, A. (2007). Astable regime in electrosprays. Physical Review E, 76, 026320.

Miao, P., \& Xiao, P. (2002). Formation of ceramic thin films using electrospray in cone-jet mode. IEEE Transactions on Industry Applications, $38,50-56$.

Mishra, S., Barton, K.I., Alleyne, A.G., Ferreira, P.M., \& Rogers, J.A. (2010). High-speed and drop-on-demand printing with a pulsed electrohydrodynamic jet. Journal of Micromechanics and Microengineering, 20, 095026.

Paine, M.D. (2009). Transient electrospray behaviour following high voltage switching. Microfluid Nanofluid, 6, 775-783.

Paine, M.D., Alexander, M.S., Smith, K.L., Wang, M., \& Stark, J.P.W. (2007). Controlled electrospray pulsation for deposition of femtoliter fluid droplets onto surfaces. Journal of Aerosol Science, 38, 315-324.

Paine, M.D., Alexander, M.S., \& Stark, J.P.W. (2007). Nozzle and liquid effects on the spray modes in nanoelectrospray. Journal of Colloid and Interface Science, 305, 111-123.

Park, J.-U., Hardy, M., Kang, S.J., Barton, K., Adair, K., Mukhopadhyay, D.K., Lee, C.Y., Strano, M.S., Alleyne, A.G., Georgiadis, J.G., Ferreira, J.G., \& Rogers, J.A. (2007). High-resolution electrohydrodynamic jet printing. Nature Materials, 6, 782-789.

Park, J.-U., Lee, J.H., Park, U., Lu, Y., \& Rogers, J.A. (2008). Nanoscale patterns of oligonucleotides formed by electrohydrodynamic jet printing with applications in biosensing and nanomaterials assembly. Nano Letters, 8, 4210-4216.

Sirringhaus, H., Kawase, T., Friend, R.H., Shimoda, T., Inbasekaran, M., \& Wu, W., et al. (2000). High resolution ink-jet printing of all-polymer transistor circuits. Science, 290, 2123-2126.

Stachewicz, U., Dijksman, J.F., Burdinski, D., Yurteri, C.U., \& Marijnissen, J.C.M. (2009a). Corrections to relaxation times in single event electrospraying controlled by nozzle front surface modification. Langmuir, 25, 2540-2549

Stachewicz, U., Yurteri, C.U., Marijnissen, J.C.M., \& Dijksman, J.F. (2009b). Stability regime of pulse frequency for single event electrospraying. Applied Physics Letters, 95, 224105.

Sutanto, E., Shigeta, K., Kim, Y.K., Graf, P.G., Hoelzle, D.J., Barton, K.L., Alleyne, A.G., Ferreira, P.M., \& Rogers, J.A. (2012). A multimaterial electrohydrodynamic jet (E-jet) printing system. Journal of Micromechanics and Microengineering, 22, 045008.

Wang, D.Z., Joyasinghe, S.N., \& Edirisinghe, M.J. (2005). High resolution print-patterning of a nano-suspension. Journal of Nanoparticle Research, 7, 301.

Yurteri, C.U., Hartman, R.P.A., \& Marijnissen, C.J.M. (2010). Producing pharmaceutical particles via electrospraying with an emphasis on nano and nanostructured particles-A review. KONA Powder and Particle Journal, 28, 91-115. 